\title{
Optical properties of a Quantum-Dot Cascade Structure
}

\author{
V.M. Apalkov and Tapash Chakraborty* \\ Max-Planck-Institut für Physik Komplexer Systeme, 01187 Dresden, Germany
}

(November 15, 2018)

\begin{abstract}
We report on our theoretical studies of the luminescence spectra of a quantum cascade laser where the quantum wells in the active regions are replaced by parabolic quantum dots. We analyze the influence of shape and size of the dots on the luminescence spectra. The emission spectra have interaction induced blueshift which increases almost linearly with increasing electron number. The blueshift is smaller for larger and non-circular dots. For large dots, shape of the emission line has weak dependence on the shape of quantum dots.
\end{abstract}

Ever since the original work on quantum cascade laser (QCL) by Faist et al. [1] 3] in 1994, the unipolar semiconductor laser based on intersubband transitions in coupled quantum wells has undergone rapid developments. QCLs created in InGaAs/AlInAs systems have achieved record high power outputs in the mid-infrared range that has the potential for wide-ranging applications [5]. Unlike in conventional lasers where it is fixed by the energy bandgap of the material, emission wavelength in a QC laser is essentially determined by the thickness of the active layers. Therefore, a suitable tailoring of the active layer thickness can produce a laser wavelength over a wide range $(3-12 \mu \mathrm{m})$ of the IR spectrum for the same material. In addition, QC lasers have much higher power than diode lasers because of the cascade scheme. In this scheme, an electron, after it has emitted a laser photon in the first active region of the device, is reinjected into the next stage that follows where it emits another photon. In this way an injected electron creates, in principle, $N_{s t}$ laser photons as it traverses the device. Here $N_{s t}$ is the number of stages. Application of an external perpendicular magnetic field on the QCL has uncovered interesting quantum oscillations in magnetotransport [8], and in future might provide magnetic tuning [9] of the laser wavelength for a given $\mathrm{QC}$ laser.

In this letter, we report on the physics of a quantum cascade structure where the quantum wells in the active regions are replaced by parabolic (symmetrical and asymmetrical) quantum dots (QD). The latter objects, popularly known as artificial atoms [10], where the electron motion is quantized in all three spatial directions, have been receiving much attention. These zero-dimensional quantum confined systems are useful for investigating the fundamental concepts of nanostructures [10 12 as well as for its vast application potentials. Important recent observations in QD transport measurements are the shell filling, half-filling dictated by Hund's rule [12,13], for circular as well as elliptical quantum dots [14,15]. On the other hand, in recent years there has been considerable progress in quantum-dot laser research [16]. Because of their discrete atom-like states, quantum-dot lasers are expected to have better performance than the quantumwell lasers [17]. A quantum-dot cascade laser (QDCL)
18 with rectangular confinement potentials in the electron plane is predicted to exhibit a large blueshift $(\sim 55$ $\mathrm{meV}$ ) in diagonal transitions (for a dot size of $10 \mathrm{~nm} \times 10$ $\mathrm{nm}$ ) when the electron number was increased from 1 to 6 in the dot. Most of the quantum dot studies to date, are however, for a parabolic confinement potential (isotropic as well as anisotropic) that turned out to be more appropriate for observing shell structure and the influence of shape and size of the dot on the electronic states. QD cascade lasers, although not yet realized, might be more efficient than the present-day quantum-well cascade lasers [19]. Knowledge of its physical properties might be useful in future development of this nanostructured light source.

The single-electron Hamiltonian for our system is

$$
\mathcal{H}^{\prime}=\frac{p_{x}^{2}}{2 m^{*}}+\frac{p_{y}^{2}}{2 m^{*}}+V_{\text {plane }}(x, y)+\frac{p_{z}^{2}}{2 m^{*}}+V_{\text {conf }}(z)
$$

where the confinement potential in the $z$-direction is

$$
V_{\text {conf }}(z)=-e F z+ \begin{cases}0 & \text { for wells } \\ U_{0} & \text { for barriers }\end{cases}
$$

with $F$ being the electric field in the $z$-direction, $m^{*}$ is energy dependent due to band non-parabolicity, $m^{*}=$ $m_{e}^{*}\left(1+2 E m_{e}^{*} \gamma / \hbar^{2}\right)$, and $U_{0}$ is the conduction band discontinuity [18]. All material parameters of our model of QDCL are given in Fig. 1, where we show one active region of the QC laser structure reported by Blaser et al. [20]. The structure emits at a wavelength of $10.5 \mu \mathrm{m}$, depending upon the electric field and temperature [20]. For our present work, details of the laser structure are not important and the most relevant system for us is the active region of Fig. 1. The confinement potential in the $x y$-plane that creates the QDs is taken to be of the form

$$
V_{\text {plane }}(x, y)=\frac{1}{2} m^{*}\left(\omega_{x}^{2} x^{2}+\omega_{y}^{2} y^{2}\right)
$$

where $\omega_{x}$ and $\omega_{y}$ are the confinement energies in the $x$ - and $y$-directions respectively, corresponding to the oscillator lengths of $l_{x}=\left(\hbar / m^{*} \omega_{x}\right)^{-1 / 2}$ and $l_{y}=$ $\left(\hbar / m^{*} \omega_{y}\right)^{-1 / 2}$. For $N$-electron system, we also take into account the Coulomb interaction between the electrons

$$
\mathcal{H}_{\text {int }}=\frac{e^{2}}{\epsilon} \sum_{i<j} \frac{1}{\left|\vec{r}_{i}-\vec{r}_{j}\right|}
$$


where $\epsilon$ is the background dielectric constant. We restrict the single electron basis by 18 lowest states and numerically obtain the eigenstates of the $N$-electron system with $N=2-9$. We analyze the shape, size and electron number dependence on the the luminescence spectra of the QDCL.

During the optical transition in the active region of a QDCL, in the initial state (before optical emission) all electrons are in the second subband. In the final state (after optical emission) one electron is in the first subband, and all other electrons are in the second subband. The intensity of optical transitions is calculated from

$$
\begin{aligned}
& \mathcal{I}_{i f}(\omega)=\frac{1}{Z} \sum_{i f} \delta\left(\omega-E_{i}+E_{f}\right) \\
& \mid \int \chi_{1}(z) z \chi_{2}(z) d z \int \Phi_{i}^{*}\left(x_{1} y_{1}, \cdots, x_{N} y_{N}\right) \\
& \times\left.\Phi_{f}\left(x_{1} y_{1}, \cdots, x_{N} y_{N}\right) d x_{1} d y_{1} \cdots d x_{N} d y_{N}\right|^{2} \\
& \times \exp \left(-\beta E_{i}\right)
\end{aligned}
$$

where $Z=\sum_{i} \mathrm{e}^{-\beta E_{i}}$ is the partition function and $\beta=$ $1 / k T$. In all our computation, we take $T=20 \mathrm{~K}$. To take into account disorder in the system we introduce the spreading of each emission line in the Lorentz form so that the final intensity is

$$
\mathcal{I}(\omega)=\int d \omega_{1} \mathcal{I}_{i f}\left(\omega_{1}\right) \frac{\Delta}{\pi\left[\Delta^{2}+\left(\omega-\omega_{1}\right)^{2}\right]} .
$$

The parameter $\Delta$ in our calculation is taken to be $\Delta=2$ meV. Shell filling in a QD is characterized by sharp peaks in the current versus the gate voltage curves in single-electron tunneling spectroscopy. The corresponding addition energy shows sharp peaks for the electron numbers $2,6,12, \ldots$ in the dot that could be interpreted as shell filling of the electronic states in the dot 12, 13. Further, there are also weak peaks in addition energy for electron numbers 4 and 9 that are explained in terms of half-filled shell structure, in accordance with Hund's rule. The addition energy is defined as the chemical potential difference $\Delta \mu_{N}=\mu(N+1)-\mu(N)$, where $\mu(N)=E(N)-E(N-1)$, and $E(N)$ is the ground state energy of the $N$ electron system.

In the quantum-dot cascade structure we have also found peaks in addition energy as a function of the number of electrons. In Fig. 2, addition energies of circular and elliptical dots are presented. For small circular quantum dots $\left(l_{x}=l_{y}=5 \mathrm{~nm}\right)$ there are sharp peaks at $N=2$ and 6 as expected. There are also peaks at $N=4$ and 9 where the total spin is equal to 1 and $3 / 2$, respectively. Because these peaks result from the interelectron interaction their strength is much smaller than those at $N=2$ and 4. The shell filling and Hund's rule peaks are also observed in a larger dot $\left(l_{x}=l_{y}=10 \mathrm{~nm}\right)$. For elliptical dots there are no peaks corresponding to the Hund's rule $(N=9)$. All peaks in this case corresponds to the shell filling at even values of electron number $(N=2,4,6$ and 8).

In Fig. 3 and Fig. 4, we present the luminescence spectra of our QC laser structures with circular and elliptic quantum dots with different number of electrons in the active region. The emission spectra for non-interaction system are shown by dotted lines. For smaller quantum dots the emission lines of the non-interacting system have some internal structure that is entirely due to the nonparabolicity. The nonparabolicity also gives the small redshift of emission line of non-interaction system. For large quantum dots there is single line for all number of electrons of non-interaction system, because non-parabolicity in this case becomes less important due to the smaller values of confinement energies $\omega_{x}$ and $\omega_{y}$. The electron-electron interaction results in a huge blueshift of the emission spectra compared to the results for non-interacting electrons. The blueshift becomes smaller for larger quantum dots and also decreases for elliptic dots. This is due to smaller interaction between the electrons when the spreading of the electron wave function in $(x, y)$ plane becomes larger. The interaction between the electrons also changes the shape of the emission line. This becomes more important for smaller quantum dots (Fig. 3). For larger quntum dots (Fig. 4) the disorder makes the emission line almost single-peaked especially for large electron numbers when the interaction between the electrons becomes smaller.

We can also see that for smaller quantum dots the change of the shape of the dots changes the shape of the emission line considerably. But for larger dots the shape of the emission line is less sensitive to the shape of the quantum dots and the line has almost the single peak both for circular and elliptic dots. Although the ground state of the electron system in the initial state obey the Hund's rule for circular dots (for $N=4$ and 9) we did not find any singularity in emission spectra due to the electron shell filling.

The intersubband luminescence peaks of the QDC laser are rather insensitive to the shell effect of the QDs in the active region. For smaller (and circular) QDs, the peaks exhibit a huge blueshift as the electron number is increased, that is entirely due to the electron-electron interaction. The blueshift is smaller for larger and noncircular dots. For large dots, shape of the emission line has weak dependence on the shape of quantum dots. We also present the addition energies of the QDCL.

We thank S. Blaser for helpful discussions on the QC lasers. We also thank P. Fulde for his support and kind hospitality in Dresden. 
* Present address: Institute of Mathematical Sciences, Chennai 600 113, India. Electronic mail: tapash@mpipks-dresden.mpg.de

[1] J. Faist, F. Capasso, D.L. Sivco, C. Sirtori, A.L. Hutchinson, and A.Y. Cho, Science 264, 553 (1994).

[2] J. Faist, F. Capasso, C. Sirtori, D.L. Sivco, A.L. Hutchinson, and A.Y. Cho, Appl. Phys. Lett. 66, 538 (1995).

[3] J. Faist, F. Capasso, C. Sirtori, D.L. Sivco, A.L. Hutchinson, M.S. Hybertsen, and A.Y. Cho, Phys. Rev. Lett. 76, 411 (1996).

[4] C. Sirtori, P. Kruck, S. Barbieri, P. Collot, J. Nagle, M. Beck, J. Faist, and U. Oesterle, Appl. Phys. Lett. 73, 3486 (1998).

[5] F. Capasso, C. Gmachl, A. Tredicucci, A.L. Hutchinson, D.L. Sivco, and A.Y. Cho, Opt. Photonics News 10, 31 (1999).

[6] C. Gmachl, F. Capasso, R. Köhler, A. Tredicucci, A.L. Hutchinson, D.L. Sivco, J.N. Baillargeon, and A.Y. Cho, IEEE Circuits \& Devices 16, 10 (2000).

[7] C.R. Webster, G.J. Flesch, D.C. Scott, J.E. Swanson, R.D. May, W.S. Woodword, C. Gmachl, F. Capasso, D.L. Sivco, J.N. Baillargeon, A.L. Hutchinson, and A.Y. Cho, Appl. Opt. 40, 321 (2001).

[8] D. Smirnov, O. Drachenko, J. Leotin, H. Page, C. Becker, C. Sirtori, V. Apalkov, and T. Chakraborty, unpublished.

[9] V.M. Apalkov and T. Chakraborty, Appl. Phys. Lett. 78, 1973 (2001).

[10] P.A. Maksym and T. Chakraborty, Phys. Rev. Lett. 65, 108 (1990); T. Chakraborty, Comments Condens. Matt. Phys. 16, 35 (1992).

[11] T. Chakraborty, Quantum Dots (North-Holland, Amsterdam, 1999).

[12] S. Tarucha, D.G. Austing, T. Honda, R. van der Hage, L.P. Kouwenhoven, Jpn. J. Appl. Phys. 36, 3917 (1997); Phys. Rev. Lett. 77, 3613 (1996).

[13] M. Rontani, F. Rossi, F. Manghi, and E. Molinari, Appl. Phys. Lett. 72, 957 (1998); T. Ezaki, N. Mori, and C. Hamaguchi, Phys. Rev. B 56, 6428 (1997); M. Fujito, A. Natori, and H. Yasunaga, ibid. 53, 9952 (1996).

[14] A.V. Madhav and T. Chakraborty, Phys. Rev. B 49 (1994) 8163

[15] D.G. Austing, S. Sasaki, S. Tarucha, S.M. Reimann, M. Koskinen, and M. Manninen, Phys. Rev. B 60 (1999) 11514.

[16] M. Grundmann, Physica E 5, 167 (2000).

[17] Y. Arakawa, and H. Sakaki, Appl. Phys. Lett. 40, 939 (1982).

[18] V.M. Apalkov and T. Chakraborty, Appl. Phys. Lett. 78, 1820 (2001).

[19] C.F. Hsu, J.S. O, P.S. Zory, and D. Botez, Proc. SPIE 3001, 271 (1997); IEEE J. Sel. Top. Quantum Electron. 5, 491 (2000); N.S. Wingreen and C.A. Stafford, IEEE J. Quantum Electron. 33, 1170 (1997).

[20] S. Blaser, L. Diehl, M. Beck, and J. Faist, Physica E 7, 33 (2000).

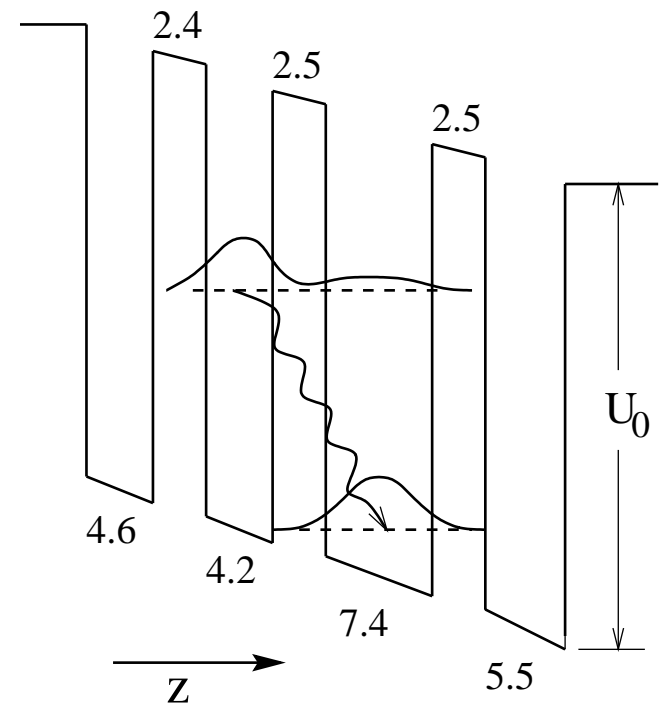

FIG. 1. Energy band diagram (schematic) of the active region of a quantum cascade laser structure under an average applied electric field of $55 \mathrm{kV} / \mathrm{cm}$ 20. Only one period of the device is shown here. The relevant wave functions (moduli squared) as well as the transition corresponding to the laser action are also shown schematically. The numbers (in $\mathrm{nm}$ ) are the well $\left(\mathrm{Ga}_{0.47} \mathrm{In}_{0.53} \mathrm{As}\right)$ and barrier $\left(\mathrm{Al}_{0.48} \mathrm{In}_{0.52} \mathrm{As}\right)$ widths. Material parameters considered in this work are: electron effective mass $m_{e}^{*}$ $\left(\mathrm{Ga}_{0.47} \operatorname{In}_{0.53} \mathrm{As}\right)=0.043 m_{0}, m_{e}^{*}\left(\mathrm{Al}_{0.48} \operatorname{In}_{0.52} \mathrm{As}\right)=0.078 m_{0}$, the conduction band discontinuity, $U_{0}=520 \mathrm{meV}$, the nonparabolicity coefficient, $\gamma_{w}=1.3 \times 10^{-18} \mathrm{~m}^{2}$ for the well and $\gamma_{b}=0.39 \times 10^{-18} \mathrm{~m}^{2}$ for the barrier.

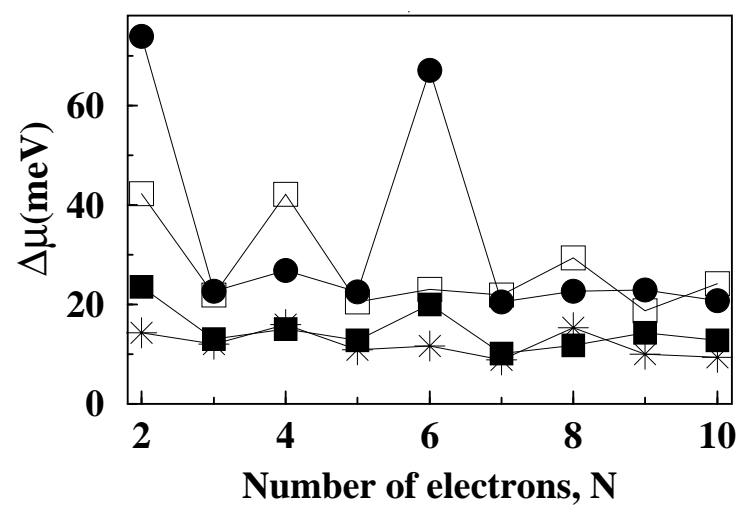

FIG. 2. Addition energies of circular and elliptical quantum dots in the QC laser. Here the sumbols indicate: • for $\left(l_{x}=l_{y}=5 \mathrm{~nm}\right), \square$ for $\left(l_{x}=5 \mathrm{~nm}, l_{y}=7 \mathrm{~nm}\right), \boldsymbol{\square}$ for $\left(l_{x}=l_{y}=10 \mathrm{~nm}\right), \star$ for $\left(l_{x}=10 \mathrm{~nm}, l_{y}=14 \mathrm{~nm}\right)$, 


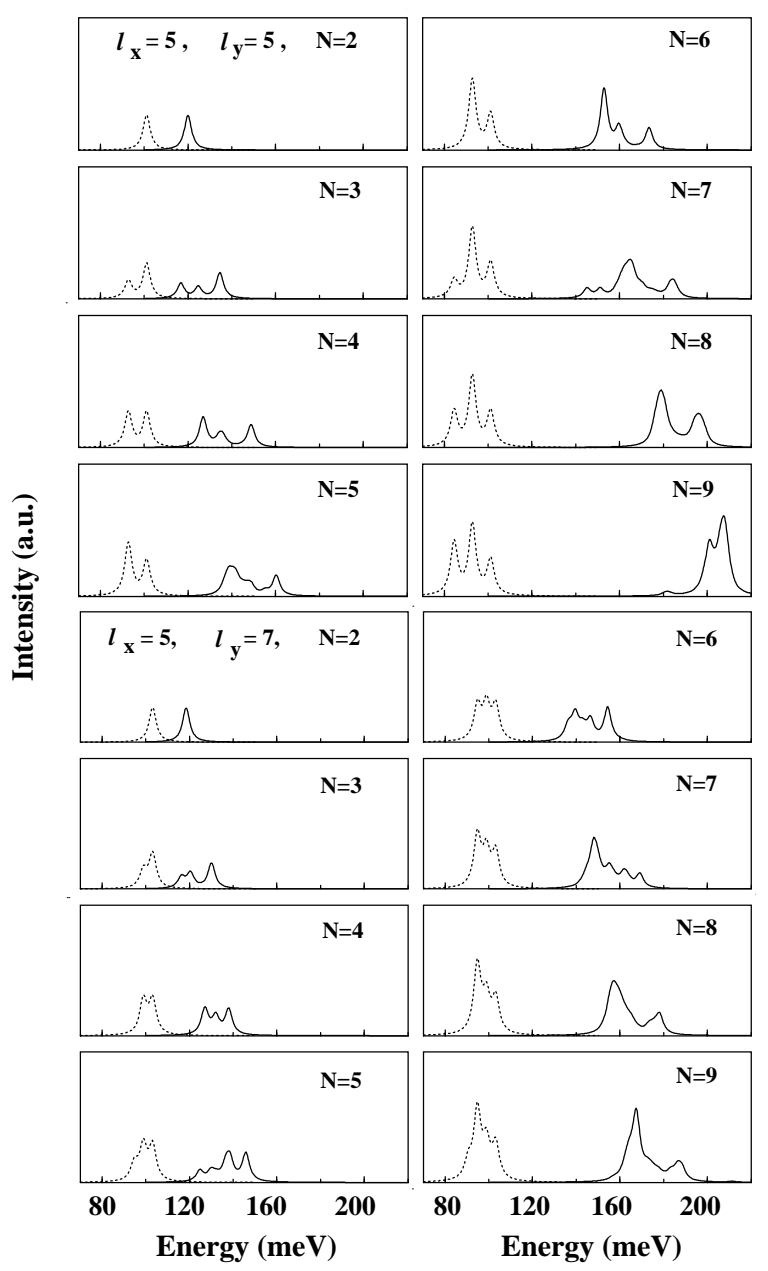

FIG. 3. Luminescence spectra of a quantum cascade laser with circular $\left(l_{x}=l_{y}=5 \mathrm{~nm}\right)$ and elliptical $\left(l_{x}=5, l_{y}=7\right.$ $\mathrm{nm})$ quantum dots containing $N=2-9$ electrons, in the active region.

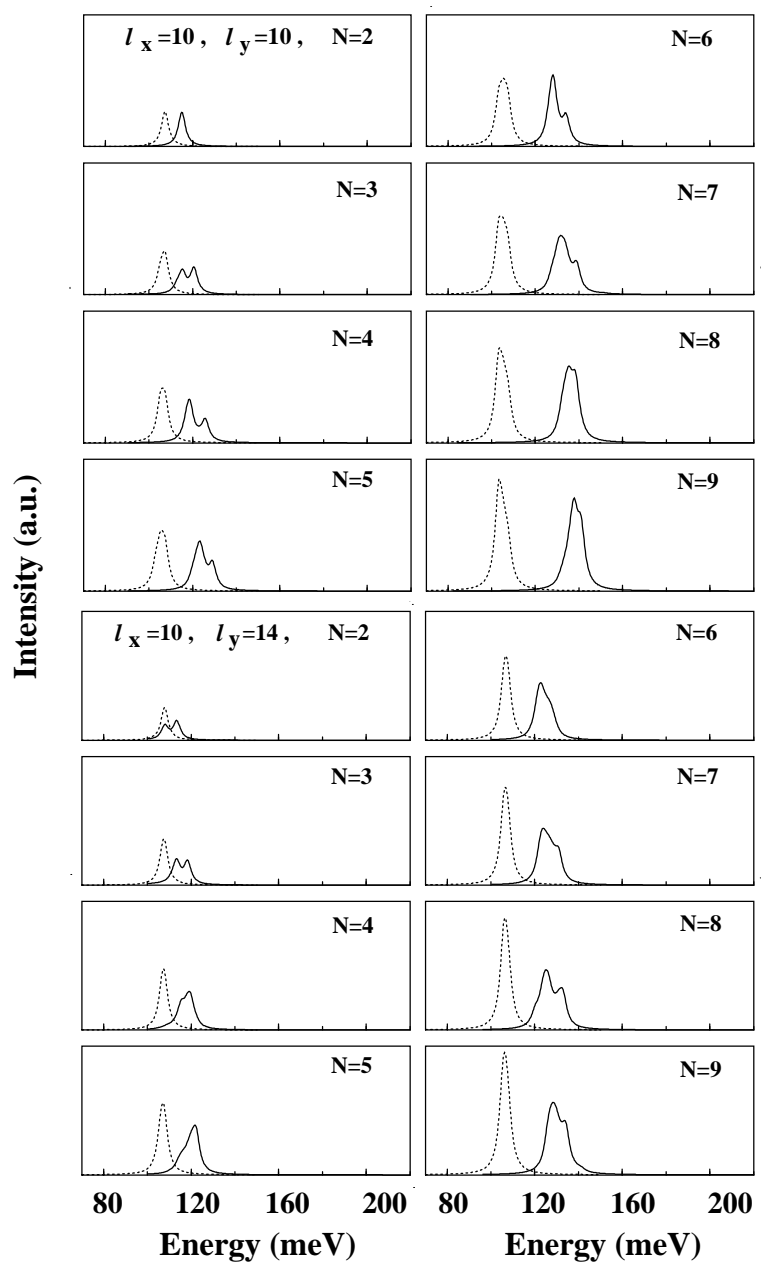

FIG. 4. Luminescence spectra of a quantum cascade laser with circular $\left(l_{x}=l_{y}=10 \mathrm{~nm}\right)$ and elliptical $\left(l_{x}=10, l_{y}=14\right.$ nm) quantum dots containing $N=2-9$ electrons, in the active region. 